\title{
Social-based Forwarding in Multi-channel Vehicular Networks
}

Link to publication record in Manchester Research Explorer

\section{Citation for published version (APA):}

Frigau, M. S. (2015). Social-based Forwarding in Multi-channel Vehicular Networks. In IEEE-ISCC Proceedings IEEE Computer Society . http://ieee-iscc.org/2015/

\section{Published in:}

IEEE-ISCC Proceedings

\section{Citing this paper}

Please note that where the full-text provided on Manchester Research Explorer is the Author Accepted Manuscript or Proof version this may differ from the final Published version. If citing, it is advised that you check and use the publisher's definitive version.

\section{General rights}

Copyright and moral rights for the publications made accessible in the Research Explorer are retained by the authors and/or other copyright owners and it is a condition of accessing publications that users recognise and abide by the legal requirements associated with these rights.

\section{Takedown policy}

If you believe that this document breaches copyright please refer to the University of Manchester's Takedown Procedures [http://man.ac.uk/04Y6Bo] or contact uml.scholarlycommunications@manchester.ac.uk providing relevant details, so we can investigate your claim.

\section{OPEN ACCESS}




\title{
Social-based Forwarding in Multi-channel Vehicular Networks
}

\author{
Matthias Sander Frigau \\ University of Manchester \\ msanderfrigau@cs.man.ac.uk
}

\begin{abstract}
Recently a lot of effort has been dedicated to maximize throughput in highly mobile multi-channel networks such as vehicular networks. Nevertheless, distributing the load as equally as possible between service channels varying in space and time is still a matter of investigation. The contributions of this paper are two-fold. Firstly, we present an adaptive multi-channel allocation mechanism (AMC) that relies on space-time discretization to fairly balance the load on service channels. Secondly, this paper investigates how the delivery performance of service updates can be enhanced by targeting vehicles which exhibit higher centrality. A Social-based Relaying Strategy (SRS) which optimizes the dissemination of service updates to create a wider situation awareness between areas of interest is introduced. This stateless strategy exploits knowledge from the social properties of the communication graph into its forwarding's weighting function. More particularly the weighting function includes a localized version of the clustering coefficient metric and the lobby index in order to select forwarding vehicles with high centrality and strong connectivity. Simulations based on a post-crash scenario demonstrate that SRS can substantially improve the delivery performance compared to GPSR-MA, a stateless geographical forwarding algorithm and slightly outperforms another socialbased forwarding strategy which only accounts for lobby index.
\end{abstract}

\section{INTRODUCTION}

Vehicular Networks is a type of mobile ad hoc network that can provide scalable and cost-effective solutions for applications such as road traffic safety, dynamic route planning, and context-aware advertisement using Vehicle-to-Vehicle (V2V) communication. Regardless of the significant research efforts VANETs are still in their infancy and have to cope up with many stringent needs such as the low market penetration rate, the large-scale network size, the quality of service delivered in a highly volatile environment, the intermittent connectivity and so on. Due to the the growing interest in Intelligent Transportation Systems, IEEE 1609.x/802.11p family of standards has been proposed to make vehicles being able to communicate on the control channel $(\mathrm{CCH})$ and the service channels $(\mathrm{SCHs})$ by periodically switching between them every fixed Synchronization Interval (SI) [1]. IEEE 802.11p also introduces WAVE Short Messages (WSMs) [2] which consist of each vehicle broadcasting small status messages which help to improve situational awareness among vehicles. However, in situations where vehicles have a large transmission range $(1000 \mathrm{~m})$ the service channel can quickly become saturated especially under high vehicular density. As a consequence this will lead to a dramatic throughput reduction and prevent the use of throughput-sensitive services on SCHs. Therefore, a major challenge in vehicular networks where vehicles are equipped with only one half-duplex radio transceiver is the dynamic allocation of channels so that the channel occupation is fairly balanced between channels when a single radio interface uses more than one $\mathrm{SCH}$. As opposed to the existing multi-channel allocation algorithms in the literature, here the objective is not to maximize the throughput but $i$ ) to increase the probability of selecting the least occupied $\mathrm{SCH}$ at a given time and point in space; $\mathrm{ii}$ ) to allow a fair access to service channels while avoiding a high reuse of the channels in time and space. Doing so will render the vehicular network more suitable for content sharing and other social applications which are delaytolerant. Thus we propose an adaptive multi-channel (AMC) allocation algorithm based on the space-time discretization of road segments in areas and zones to tackle the aforementioned objectives. This algorithm relies on one-hop periodic service updates between nodes belonging to the same area. However in some scenario, the dissemination of service updates must reach distant vehicles (e.g. belonging to another area) which render the use of forwarding mandatory. As the communication nature in vehicular networks (broadcast) and the mobility nature driven by human decisions can be seen as social behaviours, we propose Social-based Relaying Strategy (SRS), a positionbased routing protocol which utilizes localized social metrics in its forwarding decision process in order to increase the coverage of $\mathrm{SCH}$ updates outside the communication range. Social metrics are inherent to this type of networks and Social Network Analysis (SNA) appealing to solve problems such as the optimal placement of RSUs (Roadside Units), data routing and data forwarding between communities of vehicles (subgraphs). As people travel every day between home and office on the same roads at approximately the same time [3], they tend to create virtual social communities on the road or Vehicular Social Networks (VSNs) [4]. With the growing interest in large-scale and complex networks, researchers are paying more attention to the complex social behaviour of networks continuously evolving in time and space. Studies [5][6] have been conducted to highlight the importance of understanding the complex and latent behaviour of such networks. Benefits from using knowledge from the underlying network social structure have been reported in many areas, including areas such as vehicular networks [7] and public bus transportation [8]. For an ecological community, the idea of correlated interaction means that an organism of a given type is more likely to interact with another organism of the same type than with a randomly chosen member of the population [9]. Interestingly enough this concept can be incorporate to vehicular networks where drivers meet other drivers organically (e.g. work, shopping mall, arterial roads) and tend to form communities. For instance a bus has a broader coverage in space and time as it tends to have more links (high degrees) with other vehicles and longer contact durations. Thus, buses 


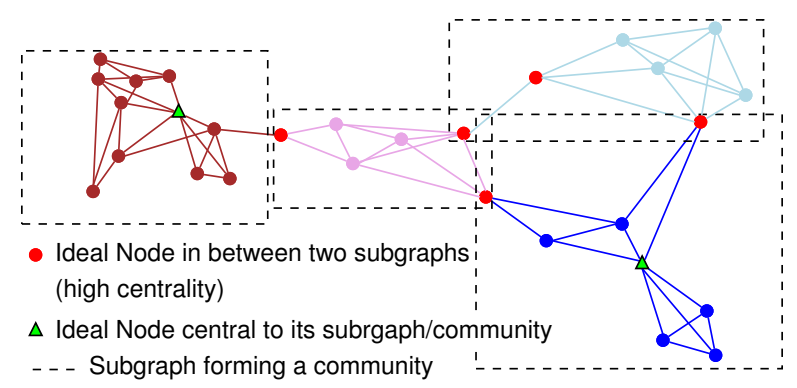

Fig. 1: Subgraphs forming communities

exhibit a higher centrality (more central to its community) which make buses good candidates for relaying information. As identifying communities or quality nodes based on their social role can require the entire topological knowledge, here we resort to a fully distributed stateless forwarding. Instead of the network-wide clustering coefficient metric, the forwarding decision process of SRS relies on the Localized Clustering Coefficient (LCC) and another localized metric called lobby index. Figure 1 shows the roles that can be played by a node with high lobby index value: it can potentially identify nodes central to their subgraphs or nodes at the border of subgraphs. As the correlation between social metrics and physical network operations is only at its infancy, this paper aims at investigating how social metrics can have an impact on geographical forwarding in a specific scenario. Here a forwarding strategy is proposed to optimize the service updates dissemination of the multi-channel allocation algorithm. This forwarding strategy called Social-based Relaying Strategy (SRS) allows vehicles to select quality relays to forward events until a vehicle in between two overlapping zones of interest is discovered. By selecting vehicles which have higher centrality and connectivity around them, this strategy ensures a broader view of the multi-channel time-space distribution. This paper investigates how the use of social metrics impact the performance delivery and channel occupation in the context of an Adaptive MultiChannel allocation algorithm (AMC). AMC embeds a TimeSpace Discretization for Multi-channel Allocation (TSD-MA) along with a new Social-based Relaying Strategy (SRS) based on geographical and social forwarding that selects quality vehicles which are more visible to other vehicles (higher centrality) and minimize the number of re-broadcasts. This forwarding strategy is also evaluated separately in the context of warning events dissemination in a post-crash scenario.

The remainder of the paper is organized as follows: In Section II existing multi-channel allocation approaches are briefly presented along with existing social-based forwarding protocols in VANETs. In Section III the time-space discretization mechanism of AMC is described. Then, we present the smart relaying strategy of AMC in Section IV. Performance evaluation and observations are reported in Section V. Finally, conclusive remarks are summarized in Section VI.

\section{RELATED WORK}

The paper targets a challenging problem which addresses two connected topics: i) multi-channel allocation and ii) socialbased forwarding in vehicular networks.
Several channel allocation algorithms have been proposed for wireless ad hoc networks to maximize the throughput. The first proposals were designed to accommodate the use of multiple channels for MANETs to avoid interferences caused by concurrent transmissions. As the basic IEEE 802.11 standard was designed only for single-channel operations while the physical layer could operate on multiple channels, the first proposals were making the assumption that mobile nodes are equipped with multiple radio interfaces, which is rather expensive [10] [11]. Other proposals have been made which are either on-demand or proactive, with clock synchronization or without, static or dynamic. In [12] authors proposed to solve the multi-channel bandwidth wastage by presenting the Multichannel MAC protocol (MMAC) which performs channel allocation at every beacon interval. The aim is to allow concurrent transmissions - one on each channel - in a particular geographical area. MMAC does not use any control channel and relies on a temporal synchronization rendez-vous point at the start of each beacon interval which consequently generates more overhead. In [13] tries to minimize the bandwidth wastage in a DSRC multi-channel environment. It relies on synchronization from RSUs and coordination such as in 802.11 PCF. It exploits the time division of $\mathrm{CCH}$ by reserving a collision-free period at the beginning of each $\mathrm{CCH}$ interval. A cognitive cluster-based multi-channel MAC protocol is proposed in [14][15] where cluster-heads are responsible of assigning the channels for cluster-members. Intra-cluster communication relies on 802.11 MAC and inter-cluster communication relies on contentionfree MAC (MMAC). The main drawback is that members in inter-clusters must use two transceivers. In [16] the channel allocation is done in function of the measured Signal-toInterference Ratio (SIR) between any pair of vehicles so that it minimizes the level of inter-node interference. They have incorporated their approach into the on-demand routing protocol AODV (AODV-SIR), which is known to be not suitable under vehicular environments. Lately in [17], the Dynamic Service-Channels Allocation (DSCA) algorithm was proposed to maximize throughput by dynamically assigning different service channels to the users. In [18] authors proposed to solve the throughput reduction in Mesh networks by considering both throughput and delay when assigning channels. While their approach is interesting it has not been designed for WAVE systems and does not consider synchronization intervals.

To reach the dream of an ubiquitous vehicular world where any driver could benefit from safety and infotainment applications in any circumstance, networking protocol design must take into consideration not only the transient state of the network (routing/neighbouring table) but also the underlying social properties formed by the communication graph. Efforts to derive interesting implications useful for the vehicular networking community has first been conducted in [5]. Authors concluded that the knowledge of the spatial and temporal characteristics of the dynamic graph can make an impact on the performances of routing protocols in VANETs. They also concluded that high centrality metrics are a good indication of quality nodes and the latent behaviour of drivers, and can be used for message ferrying in a disconnected graph to avoid re-broadcasts and reduce collisions. Even if these findings are particularly relevant to the vehicular networking community, very few social-based forwarding protocols have been proposed for vehicular networks. Social relationships and 
social roles of nodes help to quantify to which extent a node is influenced by the behaviour of other nodes in the network. This assumption led to the design of SimBeTS [19] which exploits betweenness centrality (identifies bridging capabilities of weak ties), similarity (finds nodes with same social interests as the destination) and tie strength (estimates the link availability by measuring contact duration and frequency) to make routing decisions. To this day, the most noticeable forwarding algorithms making use of social metrics in vehicular networks are graph-enabled VADD and graph-enabled GPCR [7] which include lobby index and community metrics in their forwarding decision process. More recently in [20] authors presented a social-aware forwarding protocol based on fuzzy logic (FCSA) which selects a next hop based on social centrality, social activeness and social similarity. Other socio-aware proposals have been made for Delay-Tolerant Network (DTN) forwarding in opportunistic networks. Socio-Aware Overlay [21] builds a weighted contact graph with centrality nodes based on a distributed community detection. The social role of a node has been utilized to make routing decisions for Pocket Switched Networks (PSNs) such as in BUBBLE Rap [22] which exploits rank information (betweenness centrality) and community information. In BUBBLE a node forwards packets hierarchically whether an encountered node lies in the same community as the destination and has a higher global rank than its local rank. The drawback of BUBBLE is that it tends to intensify the load of high centrality nodes. In [23] authors proposed a forwarding algorithm which forwards packets to Bluetooth-enabled mobile phones that have higher Lobby Influence (popular nodes). Their experiments show that forwarding performances (delay and packet delivery) are better than BUBBLE Rap and Epidemic Routing. In SANE [24], a social-based stateless forwarding protocol for PSNs, routing decisions rely on the assumption that nodes with similar interests tend to meet more often than nodes with different interests. For a more in-depth discussion and classification of social-based forwarding protocols for opportunistic networks, we redirect readers to the survey in [25]. As centrality metrics can be retrieved only via flooding, a method which will choke the network [26], in this paper we will consider a localized community metric called localized clustering coefficient and a centrality metric called lobby index [27] explained in more details in Section IV.

\section{Time-Space Discretization fOR Multi-CHANNEL AlLOCATION (TDS-MA)}

In this section, the time-space discretization mechanism of AMC is introduced. Then, we introduce SRS which maintains the $\mathrm{SCH}$ context through an entire zone by forwarding $\mathrm{SCH}$ updates. Firstly, TDS-MA assumes the presence of a periodical beacon exchange between vehicles so that vehicles are able to maintain a state list of SCHs through $\mathrm{CCH}$. At each Synchronization Interval (SI) a vehicle selects an unoccupied $\mathrm{SCH}$ according to the estimated load on the $\mathrm{SCH}$, its geographic zone and area of interest. In the multi-channel allocation approach proposed here, it is necessary to rely on up-todate and consistent $\mathrm{SCH}$ information in order to estimate the normalized throughput on each used $\mathrm{SCH}$.

To minimize the channel occupation on the $\mathrm{SCH}$ and allow the use of services which are throughput-sensitive, the mechanism selects opportunistically a service channel among

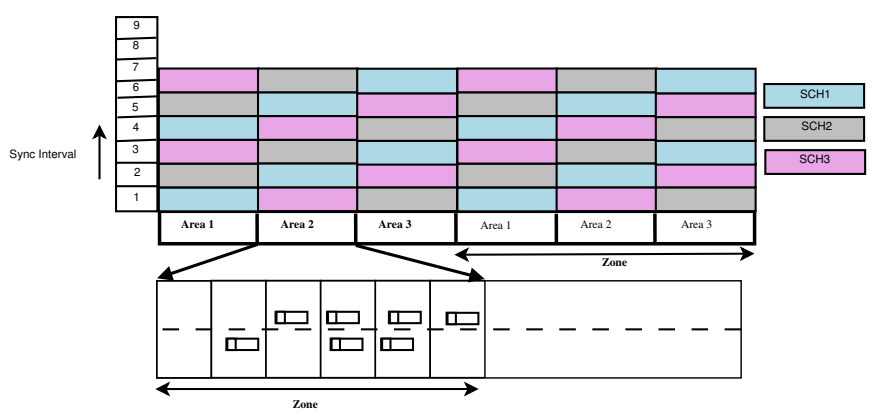

Fig. 2: TDS-MA: 3-SCHs scenario

all the six service channels. When a vehicle wants to transmit it selects an unoccupied service channel. As the state of SCHs is learned through the use of periodical beacons on the $\mathrm{CCH}$, a vehicle knows in advance which $\mathrm{SCH}$ is busy and how many vehicles are accessing it. If the list of SCHs is empty, a default $\mathrm{SCH}$ number is used. A vehicle which wants to transmit will select an unoccupied channel. It is an opportunistic mechanism which selects a $\mathrm{SCH}$ based on its state, known through the periodic exchange of beacons on $\mathrm{CCH}$. The selection of a $\mathrm{SCH}$ allows to avoid a too high reuse (in time and space) of the channels: they vary according to the time and the geographic position.

In our mechanism, depicted in Figure 2 the road is divided in zone of interest, the length of each zone of interest is $L_{Z}=2 * T_{R} * l$ where $T_{R}$ is the the transmission range of a vehicle, and $l$ is the number of lanes. Each zone is divided in areas and the number of areas $N_{A}$ equals the number of SCHs. The length of an area $L_{A}=L_{Z} / N_{A} . l$ is an important parameter and helps to configure appropriately $L_{A}$. To demonstrate the relevance of this parameter let's assume that vehicles drive on a single road with one lane with a $T_{R}$ of $200 \mathrm{~m}$. If a maximum number of six SCHs are used the length of $L_{A}$ will be of $66 \mathrm{~m}$. However on an highway, the size of an area must be increased to create a wider awareness between vehicles (an area here means a portion of the road where vehicles share the same safety measures in case of a common service or critical safety event is present). For a 3lanes road the length of an area will be $200 \mathrm{~m}$. Each area has its own channel and this channel varies with time. Each zone has a set of 6 channels which are accessed periodically according to the synchronization intervals (Sync Interval). Each vehicle manages its own allocation table which provides a default $\mathrm{SCH}$ channel number to each geographical area for a Sync interval period $(100 \mathrm{~ms})$. A GPS is used to retrieve the area in which a vehicle is.

Thanks to this geographical partitioning of the channels, vehicles will access a service according to their Area of interests where an area of interest is proportional to the transmission range divided by the number of SCHs. During each synchronization interval, if a vehicle wants to transmit data, it determines the default channel linked with its geographical position from its allocation table and checks if the channel state is not occupied. To determine if a $\mathrm{SCH}$ is occupied, a vehicle computes the following equation upon the reception of SCH information through beacons: $S C H_{B U S Y}=$ $\left(n * T_{\text {service }} * \lambda_{\text {service }}\right) * S C H I$ where $n$ is the number of 
vehicles participating in a service on one $\mathrm{SCH}, S C H I$ is the fixed-length slot of a $\mathrm{SCH}(0,46 \mathrm{~ms}), \lambda_{\text {service }}$ is the generation rate used by the service (known through beacons), and $T_{\text {service }}$ is the duration of a service packet transmitted on the $\mathrm{SCH}$, calculated as follows:

$$
T_{\text {service }}=T_{P H Y}+T_{M A C}+\frac{L_{\text {service }}}{R}+A I F S,
$$

where $T_{P H Y}$ and $T_{M A C}$ are the duration of the headers of the PHY and MAC layer respectively, $L_{\text {service }}$ is the length in bytes of a service data packet on $\mathrm{SCH}, R$ is the physical data rate, and AIFS the interframe space duration of the corresponding access class priority attached with the service. Actually, $S C H_{B U S Y}$ gives a measure of the normalized throughput on a $\mathrm{SCH}$.

To guarantee the selection of the least occupied channel, another channel is selected from the list of the SCH states if the default channel has a higher value for $T_{\text {service }}$ than another channel in the channel list state. It selects from one of its nearest neighbour Area of interest the channel which is least occupied. If it fails to find an unoccupied channel, the vehicle waits until the next synchronization period and picks the default channel of this synchronization period.

The algorithm for the selection of $\mathrm{SCH}$ works as follows:

- $\quad$ Each vehicle periodically broadcast a beacon with valuable SCH context information ( $\mathrm{SCH}$ number, Service ID in use, service generation rate) so that each neighbour can estimate the utilization of SCHs;

- Each vehicle estimates the channel utilization with updated values received from neighbours in its Area;

- Each vehicle estimates whether a $\mathrm{SCH}$ is busy or not and piggybacks $S C H_{B U S Y}$ indicator in its next beacon;

- $\quad$ Each vehicle add to its beacon payload the $\mathrm{SCH}$ state list $(\mathrm{SCH}$ state list is bound to the area the vehicle belongs to);

- At the start of the selection algorithm, if the default channel is more occupied than others, pick the least occupied;

- If the selected least occupied $\mathrm{SCH}$ will not have enough bandwidth for the service data traffic, a vehicle selects a $\mathrm{SCH}$ from a neighbouring list thanks to SRS (described in the next section).

\section{SOCIAL-BASEd RELAYING STRATEGy (SRS)}

With the AMC strategy the accuracy of the SCH state list is enhanced only if the beacon rate is not too low (below $5 \mathrm{~Hz}$ ) and if vehicles at the border of a zone successfully receive packets from both its overlapping zones. It is worth to mention that maintaining a consistent and up-to-date information about the $\mathrm{SCH}$ state list is hard to achieve in volatile environment. In the context of high-demand data provisioning the learned context through the exchange of beacons is useful in providing $\mathrm{SCH}$ state information. However, sensed and interpreted context-aware information may be uncertain or

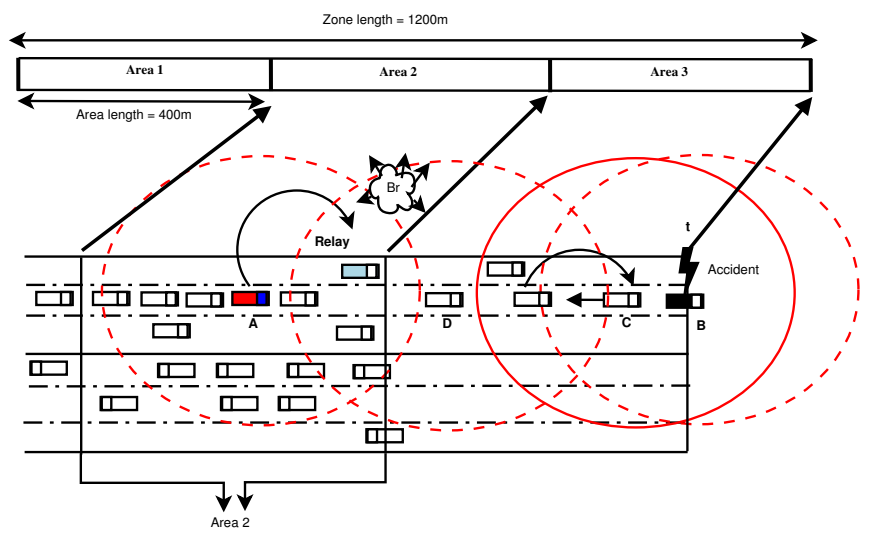

Fig. 3: Relaying strategy on an highway composed of 3-lanes road segments

obsolete which can lead to wrong decisions. Collecting and maintaining context accurately in a highly mobile environment is not easy due to vehicle's mobility and tradeoffs between context accuracy and the overhead induced. Accuracy comes at a certain cost and may result in lower network utilization but in some scenarios it is vital to ensure consistent resources availabilities which satisfy QoS [28]. As the efficiency of the multi-channel allocation of AMC depends on the accuracy of $\mathrm{SCH}$-based information received through beacons, an efficient relaying strategy is required in order to extend the visibility of an update outside of an area covered by a vehicle. Figure 3 depicts a situation where relaying is necessary to increase the awareness in-between areas so that $\mathrm{SCH}$ updates are distributed in an entire zone.

When vehicles disseminate their SCH state list (every Sync Interval) the list is not re-broadcasted thus a vehicle in zone $z_{i+1}$ might fail to receive an update from a vehicle in zone $z_{i}$ while those vehicles are part of the same driving context. In Figure 3 if vehicle D first broadcast is $\mathrm{SCH}$ state list to its 1-hop neighbours in Area 2, the neighbours of vehicle $\mathrm{D}$ in Area 3 which are in between $L_{A_{i-1}}$ and $\frac{L_{A_{i}}}{2}$ will not receive the $\mathrm{SCH}$ state list from Area 2. To remedy to this, the vehicle which is more central to Area 2 selects two quality relays which respectively are part of $\left[\right.$ Area $_{i}$, Area $\left._{i-1}\right]$, and $\left[\right.$ Area $_{i}$, Area $\left._{i+1}\right]$ where $i=2$ and these vehicle will be in charge of triggering the re-broadcast of the SCH updates. Once the central vehicle A has elected two quality relay neighbours it informs all of its neighbours of the relaying role of these two neighbours.

Figure 3 depicts a bidirectional road segment with 3-lanes in each direction, a transmission range of $200 \mathrm{~m}, 3 \mathrm{SCHs}$, so that each zone has a length of $1200 \mathrm{~m}$ divided by areas of a length of $400 \mathrm{~m}$. One of the issue in this scenario is that vehicles have a low probability to know the situation of the traffic ahead. Thus, to spread the $\mathrm{SCH}$ awareness to the entire zone we propose a social-based relaying strategy (SRS) where vehicles which exhibit a better connectivity and centrality to their area are responsible for forwarding SCH updates. SRS is initiated by the highest lobby vehicle within an area. Each high lobby vehicle takes part in the election process by computing a weighting function. A quality relay $V_{\text {relay }}$ is selected iif $V_{\text {relay }}$ it is part of two overlapping areas (a vehicle is said 
to be in between overlapping areas when it has neighbours in $A_{+1}$ (or $A_{-1}$ ) that are not neighbours of $V_{\text {relay }}$. To identify the centrality of a vehicle and the degree of connectivity of a vehicle in an area, SRS relies on two metrics:

- $\quad$ The lobby index $l i$ of a vehicle $v_{i}$ is the largest integer $k$ such that $v_{i}$ has at least $k$ neighbours with a degree of at least $k$. It gives a vehicle information about the 2-hop neighbourood. This value is calculated in our WSM Protocol by sorting the node degree of 1-hop neighbours with a Quicksort algorithm where the cost is $\mathcal{O}(n \log n): n$ is the number of one-hop neighbours degree.

- The Localized Clustering Coefficient (LCC). The clustering coefficient in its network-wide version quantifies the cliquishness of a group of connected vehicles (cluster). In it's localized version $(L C C)$, the cluster coefficient is defined as follows: if vehicle $v_{i}$ has $D_{i}(t)$ at time $t$ and there are $z_{i}(t)$ edges between its 1-hop neighbours, then: $L C C=\frac{D_{i}(t)}{z_{i}(t)}$ if $z_{i}(t)>1$. If $z_{i}(t)$ $=0$ or $1, L C C=0$. Thus, $L C C_{i}$ helps to quantify the amount of links in the neighbourhood of node $i$.

\section{A. Weighting Function}

As the awareness of the $\mathrm{SCH}$ state list within the entire zone must be increased, AMC triggers the SRS strategy at the start of every $\mathrm{SCH}$. Intermediate relays and the quality relay neighbour $V_{\text {relay }}$ - the vehicle in charge of enlarging the horizon by re-broadcasting a message - are selected by computing a weighting function. As long as none of the neighbours are part of two overlapping areas, a vehicle forwards a packet to an intermediate relay. Finally when the packet reaches a $V_{\text {relay }}$, the $\mathrm{SCH}$ state list is re-broadcasted in the next beacon.

Each next-hop relay neighbour must be the closest neighbour to the other zone, consequently the distance is part of the metric set used in the weighting function. The direction of vehicles is also an important parameter in order to know which vehicle is responsible of which area. In this way, if a vehicle is found in the middle of a zone, this vehicle will have two $V_{\text {relay }}$, one in each direction. According to [5] vehicles with high lobby index can potentially identify central nodes (good candidates to spread a message with as few re-broadcasts). From the analysis of the correlation between social metrics for different realistic vehicular traces of the city region ZentrumBellevue $^{1}$ (low and medium vehicle densities) we observed that higher the average LCC is and lower is the node connectivity which correlates with the findings in [5] as shown in Figure 4. Thus vehicles with low $L C C$ exhibit a better connectivity in their neighbourhood. For each specific entry in the neighbour table, a vehicle will select $V_{\text {relay }}$ if it has the highest weight Weighting $(j)$ :

$$
\text { Weighting }(j)=\left\{\begin{array}{r}
\left(w_{1} * \text { Distance }\right)+ \\
\left(w_{2} * \text { NormalizedDirection }\right)+ \\
\left(w_{3} \text { LobbyIndex }\right)+ \\
\left(w_{4} * \text { LCC }\right) \forall j \in v_{i}
\end{array}\right.
$$

where $w_{i}$ is the weighting factor for each criteria. The weighting factors are chosen as follows:

${ }^{1}$ Traces obtained from MMTS are available at
http://www.lst.inf.ethz.ch/research/ad-hoc/car-traces/
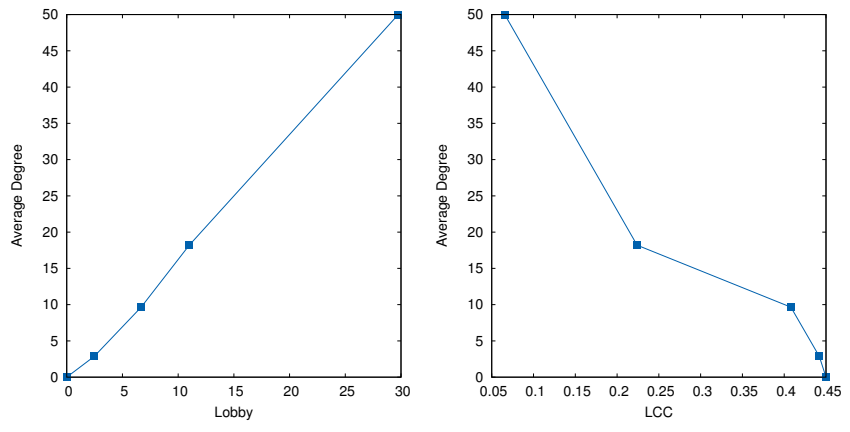

Fig. 4: (left) Average Degree vs Average Lobby Index - (right) Average Degree vs Average LCC

- Distance: euclidean distance in meters;

- NormalizedDirection: if a neighbour is identified as moving away from the vehicle computing the weighting function, the vehicle gives NormalizedDirection a value of -0.5 , if it moves closer, NormalizedDirection gets +0.5 ;

- LobbyIndex: value of the lobby index stored in the neighbour table. $\forall j \neq i$ if a neighbour $i$ satisfies $\max \left(\operatorname{lobby}_{j}\right)$ it receives +0.5 , if it satisfies $\min \left(\operatorname{lobby}_{j}\right)$ it receives -0.5 , and 0 in other cases;

- $\quad L C C$ : based on a social-network analysis of communication graphs we observed for various city center road network (details not included due to space limitation) that the average LCC has a small negative correlation with the average lobby index (Spearman's coefficient $=-0.316$ ) and node degree (Spearman's coefficient $=-0.239$ ). Thus, if the LCC is below 0.15 it helps identifying a node with a strong connectivity. A value of 0.5 identifies a half-dense neighboorhood (sparse connectivity). Consequently, if $L C C$ is above 0.15 it receives a value of +0.5 , else if it is below 0.5 it receives a value of 0 , otherwise it receives -0.5 .

The SRS algorithm is described as follows:

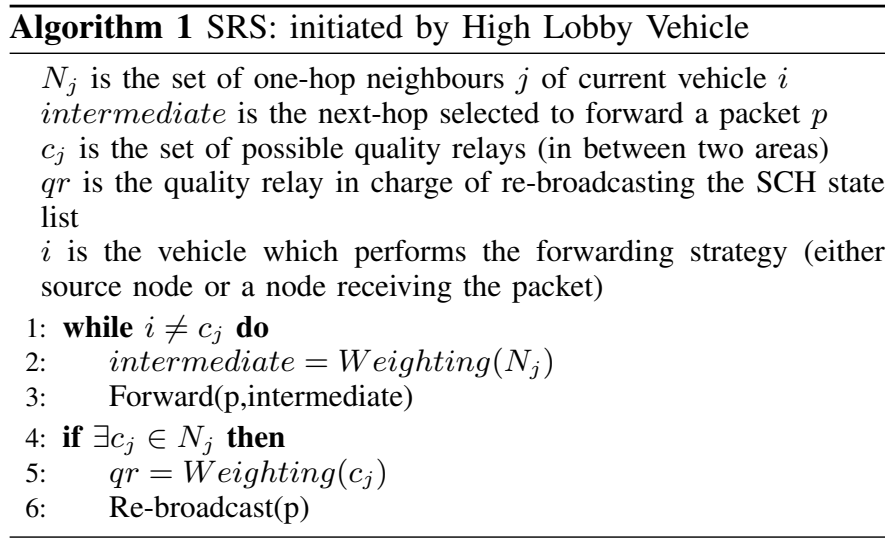

Notably, when this relaying strategy is observed from the perspective of the vehicle B victim of an accident in Figure 3, a critical post-crach safety message could be re-broadcasted to the entire zone for example, thus increasing the safetyawareness around vehicles behind which can potentially create 
a chained-collision. When such a critical event occurs, the rate of the safety messages is increased which can cause congestion for the period the critical safety event has to broadcasted. Instead another assumption is made: the critical safety message has to be relayed to the relaying neighbour in the backward direction. If vehicle B sends a WARN packet at time $t$ this packet will be relayed $r$ times until a relay found in between two overlapping areas receives it. Once the the next-hop relaying vehicle is found to part of two different areas this vehicle will re-broadcast the WARN event. As the safety-awareness is more relevant at closer distances from the accident, our strategy keeps the order of relevance by re-broadcasting first at $t+\Delta(t)$ for intermediate in $_{i}$, then at $t+\Delta(t+1)$ for intermediate $_{i+1}$, etc. until an intermediate belongs to two areas. As a result, the WARN event will be disseminated up to $r * \frac{L_{A}}{2}$ meters.

\section{PERformance Evaluation}

In this section we present the performance analysis of the proposed AMC strategy and we also study the delivery performance of SRS separately. Simulations have been conducted with the NS-3 simulator. To measure the channel occupation of AMC against different strategies (illustrated in Figure 6), a real road network map of the West University Place, Houston, TX, USA (Figure 5) has been used, which is available from the public TIGER database of the U.S. Census Bureau. Road segments of the road network are composed of two lanes. The route plans of vehicles is generated dynamically. Realistic vehicular traces have been generated with the mobility simulator VanetMobiSim. Each vehicle uses a realistic microscopic mobility model (IDM_LC) that considers mobility at a microscopic level thus describing perfectly car-to-car and intersection management. The Area and Zone information of TDS-MA are fed to NS-3 by converting the TIGER Line map with MOVE [29] and using the nod.xml and edg.xml files. The interpreted information can then be queried at simulation time by vehicles through a dedicated location service. Then, we measure the channel occupation per SCH when the 6 SCHs are used (worst case). For this set of measurements an highway scenario is assumed where all vehicles are uniformly distributed on the road with a maximum speed of $90 \mathrm{~km} / \mathrm{h}$. The highway is divided in 6 lanes, 3 lanes per direction. The distance between two lane is 5 meters and the distance between each neighbour vehicle is 10 meters. All vehicles can communicate on the basis of 1-hop broadcasting and use the 802.11p/WAVE standard with 7 channels (1 CCH and $6 \mathrm{SCHs}$ ). The transmission range is set to $200 \mathrm{~m}$. SCHs and $\mathrm{CCH}$ share the total bandwidth of $3 \mathrm{Mbps}$. The simulation settings used are depicted in Table I. The simulation time is set to 300s and 200s respectively. WSM and WSA packets use the highest priority $A C_{0}$ while service packets use the priority $A C_{3}$ which is the lowest priority in EDCA. The Nakagami-m propagation model is used with $m=1$.

\section{A. AMC evaluation}

Beforehand the performances of AMC the SCH occupation for different strategies is evaluated. The multi-channel allocation mechanism of AMC attempts to achieve a fair utilization of the service channels. Service packets are allowed to be transmitted on three service channels so that the algorithm

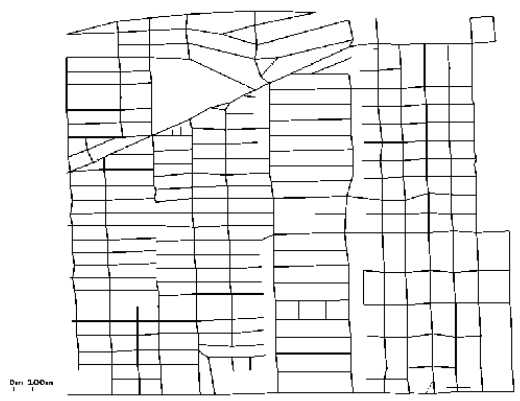

Fig. 5: Houston University Area - TIGER Map

TABLE I: Simulation settings

\begin{tabular}{|c|c|c|}
\hline PHY & Frequency & $5.9 \mathrm{Ghz}$ \\
\hline & Channel Bandwidth & OFDM 10Mhz \\
\hline & Data Rate & $3 \mathrm{Mbps}$ \\
\hline & Transmission Range & $200 \mathrm{~m}$ \\
\hline & Noise Floor & $-103 \mathrm{dBm}$ \\
\hline & Energy Detection Threshold & $-85 \mathrm{~dB}$ \\
\hline & Antenna Gain & $1 \mathrm{~dB}$ \\
\hline MAC & Slot Time, $\sigma$ & $13 \mu \mathrm{s}$ \\
\hline & Number of SCHs & $3-6$ \\
\hline Network & Switching & Alternate Access \\
\hline & Protocol & WSMP/IEEE 1609.3 \\
\hline & WSM packet size & $160 \mathrm{bytes}$ \\
\hline & WSA packet size & 200 bytes \\
\hline & REL packet size & 100 bytes \\
\hline Application & WSM frequency & $5 \mathrm{~Hz}$ \\
\hline & Service Generation Rate & $10 \mathrm{~Hz}$ \\
\hline & Service packet length & 500 bytes \\
\hline & ACK packet length & 20 bytes \\
\hline & Traffic Type & UDP Broadcast \\
\hline
\end{tabular}

will try to balance the load on three SCHs (average case). This approach is compared with three specific strategies: the first strategy is called random and refers to the fact that the number of the SCH is selected randomly among the list of all available SCHs. The second strategy is called complete and depicts the case when the list of all the SCHs is already known before the selection (ideal scenario). The fourth is called partial and corresponds to the case when only half of the SCHs are known. Figure 6 presents the channel occupation for the multi-SCH allocation. This set of measurements allows us to evaluate the efficiency of the allocation mechanism previously described. AMC shows a lower channel occupation than the random strategy. Even when the network is densely populated (90 vehicles), AMC shows clear improvements compared to the random strategy. For 90 vehicles, AMC occupies 55\% of the channel while the random strategy makes the channel completely saturated from 70 vehicles. AMC at low density, do not show any improvements. AMC performances are closed to the complete strategy. AMC performances are stable up to

TABLE II: Map and IDM_LC settings

\begin{tabular}{|c|c|}
\hline Map Area & $2387 \times 2373 \mathrm{~m}$ \\
\hline Max Speed & $15 \mathrm{~m} / \mathrm{s}$ \\
\hline Traffic light time interval & $10 \mathrm{~s}$ \\
\hline Number of lanes & 2 \\
\hline Deceleration & $0.5 \mathrm{~m} / \mathrm{s}^{2}$ \\
\hline Stay Duration & $0.5 \mathrm{~m} / \mathrm{s}^{2}$ \\
\hline
\end{tabular}




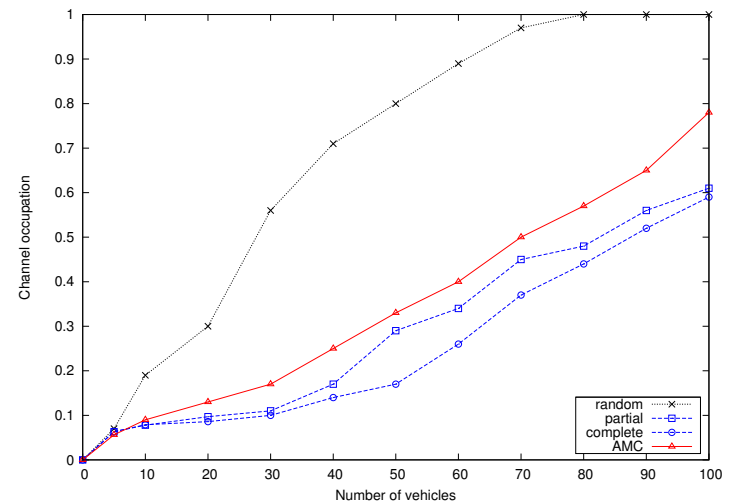

Fig. 6: Channel Occupation for different strategies

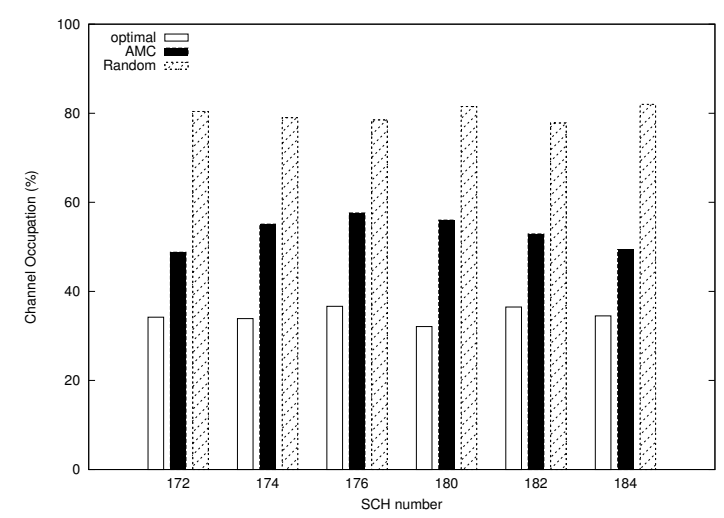

Fig. 7: Channel Occupation per SCH for different strategies

20 vehicles. However the trend of the monotonic curve depicts that the channel occupation performances slightly drop when the traffic load scales.

Figure 7 depicts the comparative performance evaluation in terms of channel occupation between three strategies. AMC presents better performances close to the complete strategy. This is due to the fact that the length of the area $(200 \mathrm{~m}$ when using $6 \mathrm{SCHs}$ ) is relatively small thus the state list is updated more frequently. If the $\mathrm{SCH}$ is occupied during the first attempt, the vehicle selects a $\mathrm{SCH}$ according to its allocation table and state list, otherwise if no $\mathrm{SCH}$ is found it relies on the state list of one of its neighbouring area via SRS.

\section{B. SRS evaluation in highway environment}

To evaluate separately SRS, a proper accident scenario has been modeled. The scenario assumes that an accident occurred at the border of a zone and that the associated WARN critical safety event must be propagated to the maximum number of vehicles behind the accident up to $r * \frac{L_{A}}{2}$ meters. SRS has been modified to re-broadcast a WARN at each intermediate relay (restricted flooding). For this scenario, we consider a Manhattan Grid where 60 vehicles have been randomly places in a 4-lanes road segment of $2400 \mathrm{~m}$ to model an highway crash situation, and 60 other vehicles have been disseminated on the rest of the grid. To validate this approach we consider static vehicles and performed simulation ten times for five different

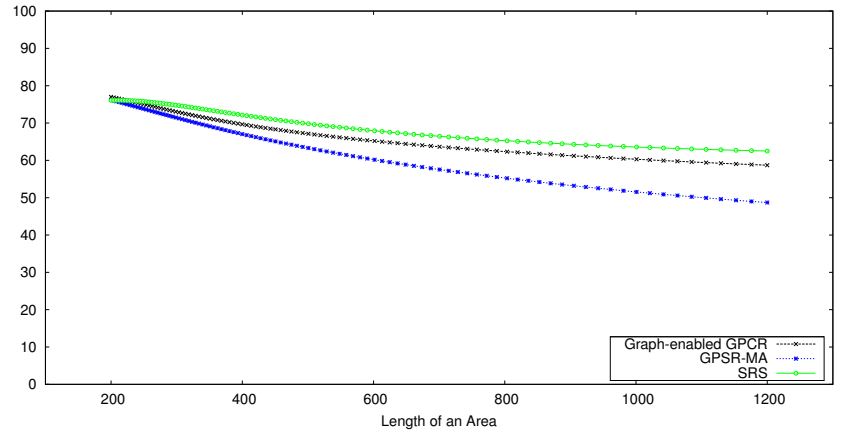

Fig. 8: Percentage (averaged over 10 runs) of neighbours receiving the WARN event for different forwarding strategies

set of vehicular traces. We plot the percentage of vehicles that successfully receive the WARN event in backward areas up to the next zone. SRS is evaluated against a stateless greedy forwarding strategy similar to GPSR-MA [30] and graphenabled GPCR [7]. Designed for city scenarios, GPCR [31] considers that the road network form a planar graph. It uses a restricted greedy forwarding to route packets along streets while routing decisions are undertaken by nodes located at intersections (coordinators). While this is not applicable to highway scenarios, graph-enabled GPCR instead elects coordinators (quality relays in this paper) with the best connectivity (high lobby index).

We decided to plot the percentage of neighbours which received successfully the WARN event with respect to different area's lengths $L_{A}$. From Figure 8 results show the same trend for the three strategies with a slight advantage for SRS for small $L_{A}$. When the length of an area is above $500 \mathrm{~m}$ the percentage of neighbours receiving the WARN event drops below $55 \%$ for GPSR-MA. The same trend is observed for SRS and graph-enabled GPCR with a small advantage for SRS. For areas of length $200 \mathrm{~m}$ and $300 \mathrm{~m}$, around $72 \%$ of vehicles receive successfully the WARN message for both strategies. When the area's length is $300 \mathrm{~m}$, the difference between GPSRMA and SRS is around 2-3\%. For larger areas of length $600 \mathrm{~m}$ and $1200 \mathrm{~m}$ the number of reached vehicles is more noticeable for SRS compared to GPSR-MA. For $L_{A}=1200 \mathrm{~m}$, while the greedy strategy is able to re-broadcast up to $46.5 \%$ of the neighbours, SRS reaches $56 \%$ of neighbours. Since the number of reachable neighbours is maximized it means that more quality relays are found with our strategy compared to the greedy strategy which accounts only for distance and direction. This is explained by the fact that LCC and lobby index together help to identify more quality relays. Interestingly enough the results for graph-enabled GPCR closely follow the results of SRS for higher $L_{A}$. Consequently, a forwarding strategy which accounts only for the lobby index achieves slightly less better than a forwarding strategy which also considers the localized clustering coefficient $L C C$ in its forwarding decisions.

\section{CONCLUSION}

This paper investigated how social metrics used in the forwarding decision process can play an impact on the network performance. We first presented TSD-MA which relies on the discretization of time and space in order to fairly balance the 
load between SCHs in a given region of space and time. To coordinate the multi-channel allocation, $\mathrm{SCH}$ state lists are maintained and disseminated to neighbours. To enlarge the horizon and cover an entire zone, the $\mathrm{SCH}$ state lists must be forwarded. Consequently we have introduced a Socialbased relaying strategy to disseminate effectively the $\mathrm{SCH}$ state list between neighbouring areas belonging to the same zone. For each area, quality relays are elected to re-broadcast the $\mathrm{SCH}$ state list. Each vehicle uses a weighting function which takes into account the influence that a node has over others to re-broadcast information to the maximum number of distant neighbours. AMC demonstrated that under medium vehicular density and high mobility, because of the presence of SRS, AMC still achieves to balance the load as vehicles receive more accurate context information about $\mathrm{SCH}$ states in the neighbouring areas. Inspired by the latter strategy, we have evaluated the performance delivery of SRS in the context of a critical safety message dissemination to avoid chained collisions after an accident occurred. Compared to GPSR-MA, SRS is able to inform up to $15 \%$ more vehicles for large $L_{A}$ than GPSR-MA, and up to $3 \%$ more vehicles in average than a social-based forwarding algorithm only based on lobby index. As future work, we plan to compare SRS performance against several existing flooding techniques.

\section{REFERENCES}

[1] IEEE, "Ieee 1609.4 - ieee standard for wireless access in vehicular environments (wave) - multi-channel operation," IEEE, September 2010.

[2] — "Ieee 1609.3-2010 - ieee standard for wireless access in vehicular environments (wave) - networking services," IEEE, 2010.

[3] R. Fei, K. Yang, and X. Cheng, "A cooperative social and vehicular network and its dynamic bandwidth allocation algorithms," in Computer Communications Workshops (INFOCOM WKSHPS), 2011 IEEE Conference on, April 2011.

[4] S. Smaldone, L. Han, P. Shankar, and L. Iftode, "Roadspeak: enabling voice chat on roadways using vehicular social networks," in Proceedings of the 1st Workshop on Social Network Systems. ACM, 2008.

[5] G. Pallis, D. Katsaros, M. Dikaiakos, N. Loulloudes, and L. Tassiulas, "On the structure and evolution of vehicular networks," in IEEE International Symposium on Modeling, Analysis Simulation of Computer and Telecommunication Systems, 2009. MASCOTS '09., 2009.

[6] A. Papadimitriou, D. Katsaros, and Y. Manolopoulos, "Social network analysis and its applications in wireless sensor and vehicular networks," in Next Generation Society. Technological and Legal Issues, ser. Lecture Notes of the Institute for Computer Sciences, Social Informatics and Telecommunications Engineering. Springer Berlin Heidelberg, 2010, vol. 26.

[7] N. Loulloudes, G. Pallis, and M. D. Dikaiakos, "The dynamics of vehicular networks in urban environments," arXiv preprint arXiv:1007.4106, 2010.

[8] X.-H. Yang, G. Chen, B. Sun, S.-Y. Chen, and W.-L. Wang, "Bus transport network model with ideal n-depth clique network topology," Physica A: Statistical Mechanics and its Applications, vol. 390, no. 23 , 2011.

[9] S. Okasha, "Altruism, group selection and correlated interaction," The British journal for the philosophy of science, vol. 56, no. 4, pp. 703725,2005

[10] A. Nasipuri, J. Zhuang, and S. R. Das, "A multichannel csma mac protocol for multihop wireless networks," in Wireless Communications and Networking Conference, 1999. WCNC. 1999 IEEE. IEEE, 1999, pp. 1402-1406.

[11] S.-L. Wu, C.-Y. Lin, Y.-C. Tseng, and J.-P. Sheu, "A new multi-channel mac protocol with on-demand channel assignment for multi-hop mobile ad hoc networks," in Parallel Architectures, Algorithms and Networks, 2000. I-SPAN 2000. Proceedings. International Symposium on. IEEE, 2000, pp. 232-237.
[12] J. So and N. H. Vaidya, "Multi-channel mac for ad hoc networks: Handling multi-channel hidden terminals using a single transceiver," in Proceedings of the 5th ACM International Symposium on Mobile Ad Hoc Networking and Computing, ser. MobiHoc '04, 2004.

[13] T. K. Mak, K. P. Laberteaux, and R. Sengupta, "A multi-channel vanet providing concurrent safety and commercial services," in Proceedings of the 2Nd ACM International Workshop on Vehicular Ad Hoc Networks, ser. VANET '05. ACM, 2005.

[14] X. Zhang, H. Su, and H.-H. Chen, "Cluster-based multi-channel communications protocols in vehicle ad hoc networks," Wireless Communications, IEEE, vol. 13, no. 5, October 2006.

[15] H. Su and X. Zhang, "Clustering-based multichannel mac protocols for qos provisionings over vehicular ad hoc networks," Vehicular Technology, IEEE Transactions on, vol. 56, no. 6, pp. 3309-3323, Nov 2007.

[16] P. Fazio, F. De Rango, C. Sottile, and C. Calafate, "A new channel assignment scheme for interference-aware routing in vehicular networks," in Vehicular Technology Conference (VTC Spring), 2011 IEEE 73rd, May 2011, pp. 1-5.

[17] S. Park, Y. Chang, F. Khan, and J. Copeland, "Dynamic servicechannels allocation (dsca) in vehicular ad-hoc networks," in Consumer Communications and Networking Conference (CCNC), 2013 IEEE, January 2013.

[18] Y. Ding, K. Pongaliur, and L. Xiao, "Channel allocation and routing in hybrid multichannel multiradio wireless mesh networks," Mobile Computing, IEEE Transactions on, vol. 12, no. 2, pp. 206-218, Feb 2013.

[19] E. M. Daly and M. Haahr, "Social network analysis for information flow in disconnected delay-tolerant manets," Mobile Computing, IEEE Transactions on, vol. 8, no. 5, pp. 606-621, 2009.

[20] X. Gu, L. Tang, and J. Han, "A social-aware routing protocol based on fuzzy logic in vehicular ad hoc networks," in High Mobility Wireless Communications (HMWC), 2014 International Workshop on, Nov 2014, pp. 12-16.

[21] E. Yoneki, P. Hui, S. Chan, and J. Crowcroft, "A socio-aware overlay for publish/subscribe communication in delay tolerant networks," in Proceedings of the 10th ACM Symposium on Modeling, analysis, and simulation of wireless and mobile systems. ACM, 2007, pp. 225-234.

[22] P. Hui, J. Crowcroft, and E. Yoneki, "Bubble rap: Social-based forwarding in delay-tolerant networks," Mobile Computing, IEEE Transactions on, vol. 10, no. 11, pp. 1576-1589, Nov 2011.

[23] S. Khan, R. Mondragon, and L. Tokarchuk, "Lobby influence: Opportunistic forwarding algorithm based on human social relationship patterns," in Pervasive Computing and Communications Workshops (PERCOM Workshops), 2012 IEEE International Conference on, March 2012.

[24] A. Mei, G. Morabito, P. Santi, and J. Stefa, "Social-aware stateless forwarding in pocket switched networks," in Infocom, 2011 Proceedings Ieee. IEEE, 2011, pp. 251-255.

[25] M. R. Schurgot, C. Comaniciu, and K. Jaffres-Runser, "Beyond traditional dtn routing: social networks for opportunistic communication," arXiv preprint arXiv:1110.2480, 2011.

[26] P. Muhlethaler, A. Laouiti, and Y. Toor, "Comparison of flooding techniques for safety applications in vanets," in Telecommunications, 2007. ITST '07. 7th International Conference on ITS, June 2007.

[27] A. Korn, A. Schubert, and A. Telcs, "Lobby index in networks," Physica A: Statistical Mechanics and its Applications, vol. 388, no. 11, 2009.

[28] Y. Huang, S. Mohapatra, Q. Han, and N. Venkatasubramanian, "Qos aware resource discovery in mobile environments," The Handbook of Mobile Middleware, 2009.

[29] F. Karnadi, Z. H. Mo, and K.-C. Lan, "Rapid generation of realistic mobility models for vanet," in Wireless Communications and Networking Conference, 2007. IEEE WCNC 2007., March 2007.

[30] F. Granelli, G. Boato, D. Kliazovich, and G. Vernazza, "Enhanced gpsr routing in multi-hop vehicular communications through movement awareness," Communications Letters, IEEE, vol. 11, no. 10, October 2007.

[31] C. Lochert, M. Mauve, H. Fussler, and H. Hartenstein, "Geographic routing in city scenarios," SIGMOBILE Mob. Comput. Commun. Rev., vol. 9, January 2005. 